\title{
Uma Abordagem para Melhoria do Processo de Software baseada em Medição
}

\author{
Renata Teles Moreira ${ }^{1}$, Geovane Nogueira Lima ${ }^{1}$, Breno Batista Machado ${ }^{1}$, Weslei \\ de Tarso Marinho ${ }^{1}$, Alexandre Vasconcelos ${ }^{1}$, Ana Cristina Rouiller ${ }^{2}$ \\ ${ }^{1}$ Centro de Informática - Universidade Federal de Pernambuco (UFPE) \\ Recife - PE - Brasil \\ ${ }^{2}$ Universidade Federal Rural de Pernambuco (UFRPE) \\ Recife - PE - Brasil \\ $\{$ rtm, gnl, bbm, watm, amlv\}@cin.ufpe.br, anarouiller@gmail.com
}

Resumo. Este artigo apresenta uma abordagem para Melhoria de Processo de Software, definida através da simplificação e adaptação do DMAIC, tendo como foco principal a melhoria baseada na medição dos processos. A abordagem visa trabalhar os processos prioritários para a organização $e$ relevantes para os clientes, utilizando ciclos de melhoria menores, com intuito de obter resultados rápidos e significativos.

Abstract. This paper presents an approach for Software Process Improvement, defined through the simplification and adaptation of DMAIC, being the main focus the improvement based on processes measurement. Our approach addresses the priority processes for the organization and the relevant processes for the clients, using less cycles of improvement, with intention of obtaining quick and significant results.

\section{Introdução}

Nas ultimas duas décadas, Melhoria de Processo de Software (MPS) tem se tornado um importante tópico para pesquisas acadêmicas na área de Engenharia de Software, assim como para organizações de software que estão tentando ser competitivas neste desafiante mercado global (Serrano et al, 2006). Um dos principais objetivos da MPS é produzir software de qualidade, no prazo, dentro do orçamento e, de acordo com as especificações do cliente (Rocha et al, 2001). A qualidade em MPS é baseada na premissa que processos maduros e capazes geram produtos de qualidade. Esta teoria é fundamentada principalmente nos trabalhos desenvolvidos nas décadas passadas por Shewhart (1931), Deming (1986), Juran (1988) e Humphrey (1995).

No cenário atual, a MPS tem sido baseada, principalmente, em modelos, normas e metodologias como CMMI (Chrissis et al, 2007), ISO/IEC 15504 (ISSO/IEC 15504, 2003), ISO 9000 (NBR-ISO 9000, 2000), ISO/IEC 12207 (NBR-ISO/IEC 12207, 1998), MR-MPS (SOFTEX, 2007) e Seis Sigma (Tayntor, 2003). Estes modelos são considerados um conjunto de boas práticas para o desenvolvimento de projetos, produtos e serviços.

Dentre estes modelos, o CMMI tem sido o mais utilizado mundialmente por organizações em programas de MPS. No cenário nacional, o modelo MPS.BR tem cada vez mais ganhado importância e reconhecimento por parte da indústria local. Este 
modelo surgiu com o objetivo de viabilizar a implantação de MPS de forma adequada ao perfil das empresas nacionais, com foco principal nas micro e pequenas empresas.

É importante observar que a MPS envolve inúmeros desafios e dificuldades intrínsecas, os quais contribuem para o insucesso de várias iniciativas (Santana, 2007). De acordo com Salviano (2006), existe uma estatística informal, que circula pela comunidade, que de cada três iniciativas de melhoria de processo apenas uma tem sucesso. Segundo estudos (Santana 2007; Dybã, 2003; Dybã, 2005), o insucesso destas iniciativas pode ocorrer por vários motivos, considerados fatores críticos para MPS, tais como: falta de apoio e comprometimento da alta administração, restrições de tempo e recurso, falta de uma metodologia apropriada e resistência à mudanças.

Dentre os fatores críticos para MPS, um dos pontos observados é a necessidade de uma abordagem apropriada. Como exemplos de abordagens podemos citar: o modelo IDEAL, o Ciclo de Melhoria da ISO/IEC 15504, o PRO2PI e o modelo DMAIC (Harry e Schroeder, 1998; Pande et al, 1998; Eckes, 2001). As três primeiras são abordagens propostas para o contexto de MPS, enquanto o DMAIC é um método originalmente definido para implementação da metodologia Seis Sigma nos processos de manufatura das organizações (Tayntor, 2003).

Embora o DMAIC não seja dirigido às organizações de software, empresas desta indústria têm investido na implementação das suas técnicas para melhoria dos seus processos de software (Siviy et al, 2008). Estas iniciativas têm sido motivadas, especialmente, pelo foco do DMAIC na melhoria de processo de forma quantitativa, através de medições e controle estatístico do processo.

Goethert and Hayes (2001) afirmam que a preocupação em medição é um fator essencial para facilitar uma iniciativa de melhoria de processo de software bem sucedida. A medição não é apenas uma forma eficiente para identificar, reconhecer e avaliar as melhorias no processo, ela também tem uma importância crucial para apoiar e direcionar essas melhorias (Florac et al, .

Atualmente a utilização do DMAIC para MPS tem se restringido a apoiar as organizações na implementação dos altos níveis de maturidade dos modelos de referência, não sendo observada, ainda, a sua utilização para a melhoria de forma independente desses modelos. De acordo com Siviy et al (2008) muitas destas restrições se devem ao grau de dificuldade intrínseco associada à implantação desta metodologia, somada à dificuldade de sua adaptação ao contexto de software.

Desta forma, acredita-se que uma metodologia mais simples e direcionada ao contexto de software, possa contribuir com aplicação da melhoria de processo baseada no desempenho dos processos de uma organização de software independente do seu nível de maturidade e dos modelos adotados por ela.

Neste contexto, este artigo apresenta a abordagem Modus ${ }^{1}$, uma abordagem baseada nos conceitos de medição e análise de desempenho do processo para implementação de MPS. Esta abordagem foi definida através da simplificação e adaptação do DMAIC e visa trabalhar os processos prioritários para a organização e relevantes para os clientes, utilizando ciclos de melhoria mais curtos, com o intuito de obter resultados rápidos e significativos.

\footnotetext{
${ }^{1}$ A palavra Modus vem do latin e significa medida, limites, maneira.
} 


\section{Abordagem Modus}

A principal proposta com o desenvolvimento da abordagem Modus é viabilizar às organizações a identificação de oportunidades de melhoria pontuais nos processos. Essas oportunidades são identificadas através da medição e análise do comportamento e desempenho do processo, sendo tratadas, avaliadas e validadas progressivamente de forma mensurável. Durante a definição da abordagem buscou-se considerar os seguintes pontos:

- Independência de pré-requisitos quanto à existência de um processo de medição definido;

- Independência em relação aos modelos e normas de referência para MPS;

- Foco em melhorias pontuais do processo;

- Facilidade de compreensão e implementação;

- Aplicável em organizações de baixa maturidade;

- Obtenção de resultados rápidos e mensuráveis.

A abordagem Modus foi elaborada tomando a metodologia DMAIC como principal referência, visando adaptá-la ao contexto das organizações de software e tendo em vista as características acima relacionadas. Desta forma, a abordagem pode ser vista como uma simplificação e adaptação do DMAIC ao contexto particular de desenvolvimento de software. Ainda foi considerado, como fundamentação teórica, as principais abordagens e modelos para MPS e metodologias e técnicas para medição em software.

Outro ponto importante na definição da abordagem Modus foi a observação do comportamento de programas de melhoria nas organizações. Estas observações foram realizadas, por meio de experiência prática dos autores na implementação de modelos da qualidade, principalmente o MR-MPS e, através de análise de relatos de experiência e estudos de caso de implementação de MPS. O universo das empresas observadas constituiu-se principalmente de pequenas e médias organizações, que se encontravam em baixos níveis de capacidade em relação aos principais modelos

\subsection{Visão Geral da Abordagem Modus}

A abordagem Modus está fortemente baseada nos conceitos de medições. Esta envolve a aplicação de métodos estatísticos para analisar o comportamento dos processos e, a partir daí, fornecer subsídios para sua melhoria, incluindo a análise de possíveis causas de defeitos, aplicação de ações corretivas, implantação e avaliação das melhorias.

A abordagem visa fornecer, por meio da análise de dados obtidos em medições, uma visão objetiva do comportamento dos processos, permitindo a compreensão de seu status, suas variações de desempenho e qualidade e o grau de alcance dos objetivos de negócio da organização e do atendimento às necessidades dos clientes. Normalmente as oportunidades de melhoria são identificadas através da observação de processos que apresentam desempenho abaixo do esperado, conforme os objetivos de negócio e requisitos do cliente.

Para a aplicação da abordagem, os processos que consomem recursos significativos ou que apresentam relação com a qualidade do produto devem ser priorizados. Estes processos, comumente chamados de processos críticos, concentram 
pontos com maior potencial de retorno com a melhoria. Também é importante observar que mudanças radicais devem ser evitadas no processo para se poder utilizar a base histórica de medidas na análise do comportamento dos processos.

Visando uma abordagem de fácil aplicação e compreensão, buscou-se utilizar, prioritariamente, as ferramentas de medição, análise e controle que fossem mais simples. Embora simples, quando aplicadas de forma correta, as ferramentas provêm resultados significativos e confiáveis. Tais ferramentas não exigem um conhecimento muito aprofundado em estatística e nem a aquisição de softwares específicos para sua aplicação, sendo possível implementá-las, com pouco esforço, através de planilhas eletrônicas.

A abordagem Modus é composta por quatro fases. A primeira fase é uma fase de conhecimento dos objetivos da organização e inicialização do programa de melhoria dentro da empresa e acontecerá uma única vez. A segunda fase é para definir o escopo do Ciclo de Melhoria na organização, ou seja, quais os processos irão ter seu desempenho melhorado através do Ciclo de Melhoria da abordagem Modus. O Ciclo de Melhoria é a fase mais importante e onde as mudanças acontecem. Esta fase é iterativa e só termina quando o processo tiver alcançado o desempenho desejado. A última fase é a de Controle, onde os processos melhorados passam a ser monitorados quantitativamente a fim de garantir o desempenho alcançado.

A Figura 1 fornece uma visão geral da abordagem e suas fases.

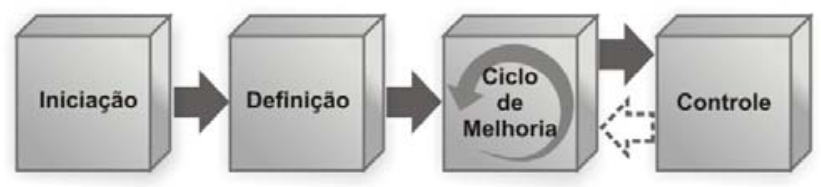

Figura 1 - Fases da Abordagem Modus

Cada fase é subdividida em atividades que são detalhadas para prover uma orientação efetiva aos responsáveis de como aplicá-las. Cada fase é composta pelo seu objetivo e uma ou mais atividades constituídas de um propósito, entradas, saídas, responsável, participantes passos e orientações gerais.

Os papéis envolvidos na implantação da abordagem Modus vão desde a alta direção da organização até a equipe de desenvolvimento. Para que o programa de melhoria tenha resultados positivos é importante que toda a organização esteja envolvida e comprometida com sua execução. Para a aplicação da abordagem não há obrigatoriedade de se ter Black Belts ou mesmo Green Belts ${ }^{2}$ na liderança, como é apresentado na metodologia DMAIC. Por serem de cunho simples e diretamente relacionado aos processos da organização, é necessário apenas um conhecimento básico sobre estatística e treinamento na abordagem. Neste caso, o que se torna mais relevante é o entendimento relacionado ao negócio da organização e técnicas de gestão, sendo, portanto, indicado que alguém da gerência lidere o programa. O tamanho da equipe para aplicação da abordagem pode variar de acordo com a necessidade do problema.

\footnotetext{
${ }^{2}$ Black Belts e Green Belts são os especialistas na metodologia Seis Sigma, responsáveis por desenvolver e orientar os times de melhoria na utilização do DMAIC.
} 


\subsection{Fases da Abordagem}

Esta seção apresenta uma descrição geral de cada fase da abordagem Modus. A abordagem completa e detalhada está disponível para consulta através do site: http://www.cin.ufpe.br/ processos/modus.

\section{Fase 1: Iniciação}

A fase Iniciação, como ilustrado na Figura 2, tem como objetivo formalizar o início do programa de melhoria dentro da organização. Nesta fase, o contexto da organização é conhecido, os objetivos iniciais da melhoria e a equipe são definidos, o comprometimento com o programa é obtido e o programa é lançado na organização. A atividade desta fase é descrita a seguir:

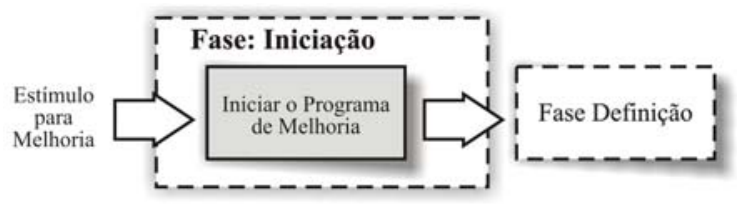

Figura 2 - Atividade da Fase Iniciação

Iniciar o Programa de Melhoria - O objetivo dessa atividade é formalizar o interesse da organização em iniciar um programa de melhoria do processo de software. Neste momento os responsáveis pelo programa de melhoria são definidos, o contexto da organização é analisado e o comprometimento com o programa é obtido com a alta direção. Nesta atividade uma abertura oficial do programa de melhoria é realizada na organização.

\section{Fase 2: Definição}

A fase de "Definição", ilustrada na Figura 3, tem como objetivo identificar as necessidades de melhoria com base nos objetivos de negócio e necessidades dos clientes.

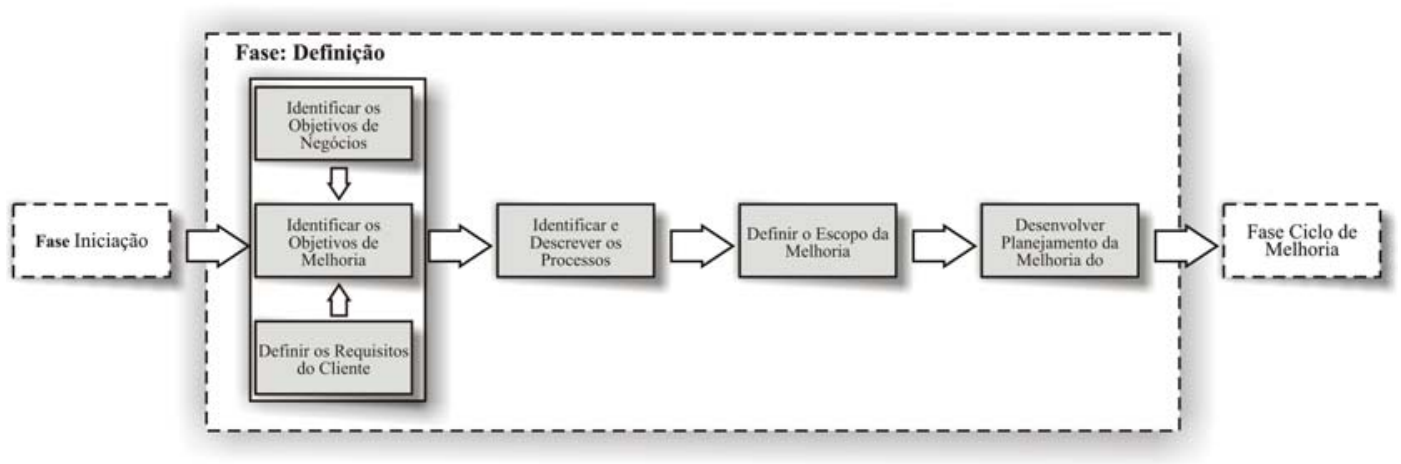

Figura 3 - Atividades da Fase Definição

Nesta fase os processos da organização são identificados e o seu estado atual é documentado através de descrições e/ou mapas de processo. Posteriormente os processos críticos da organização são selecionados e priorizados para a implementação do Ciclo de Melhoria, caracterizando assim o escopo da melhoria.

Um planejamento é elaborado para a implementação das melhorias no processo, considerando recursos, responsabilidades, cronograma, metas e riscos associados ao 
programa de melhoria. Esta fase pode ser revista na organização periodicamente, incluindo ou retirando processos do escopo da melhoria conforme necessário. As atividades da fase Definição são descritas a seguir:

Identificar os Objetivos de Negócio - Durante esta atividade o Gestor do Programa promove reuniões com os níveis gerenciais apropriados para a definição dos objetivos de negócio da organização. Esses objetivos devem estar atrelados à visão e missão que a organização tem definidas.

Identificar os Objetivos de Melhoria - Nesta atividade devem-se identificar os Objetivos de Melhoria do Programa de forma a satisfazer os objetivos de negócio, considerando as oportunidades de melhoria dos processos organizacionais. Depois de identificados, os objetivos de melhoria devem ser validados com a alta direção e registrados em local visível a todos os colaboradores da organização.

Definir os Requisitos do Cliente - Nesta atividade os principais clientes da organização são identificados e as suas necessidades relacionadas ao produto ou serviço, objeto da melhoria, são definidos e registrados.

Identificar e Descrever os Processos - O objetivo desta atividade é identificar e descrever todos os processos da organização, alvos potenciais da melhoria. Para cada processo deve ser determinado um time de trabalho, o qual deve ser composto por pessoas envolvidas no processo em questão. A descrição dos processos irá ajudar na identificação dos problemas e de quais processos deverão ser alterados como parte da resolução destes.

Definir o Escopo da Melhoria - Nesta atividade o escopo da melhoria é definido, através da priorização e escolha dos processos, de forma a maximizar o retorno do investimento em melhoria. Para tal devem ser considerados os requisitos dos clientes e objetivos de melhoria frente à relação de custo benefício da melhoria associada ao processo.

Desenvolver Planejamento da Melhoria do Processo - Nesta atividade o planejamento do Ciclo de Melhoria do Processo é realizado considerando recursos, responsabilidades, cronograma, metas e riscos associados ao programa de melhoria.

\section{Fase 3: Ciclo de Melhoria}

A fase "Ciclo de Melhoria”, exibida na Figura 4, envolve o refinamento dos processos pré-selecionados, identificando as medidas que serão necessárias para conhecer o comportamento do processo, a elaboração de um procedimento de medição, bem como a identificação e proposição de melhoria para o processo, e ainda, a validação destas. As atividades desta fase são realizadas continuamente até que o processo obtenha o desempenho desejado.

O primeiro "Ciclo de Melhoria" de um determinado processo tem como objetivo principal conhecer e entender o comportamento e desempenho do mesmo, portanto recomenda-se que ele passe por todas as atividades da fase "Ciclo de Melhoria". Os próximos ciclos estão relacionados à proposição de melhorias e validação destas com base no desempenho dos processos. Uma vez que o processo atinge o desempenho desejado no ciclo de melhoria, este deverá ser colocado sobre o controle na próxima fase da abordagem. 
É importante ressaltar que, se a organização não conhece o comportamento e desempenho do processo, é natural que o primeiro ciclo demande um esforço significativamente maior que nos demais ciclos, uma vez que este envolve detalhar o mapa do processo e identificar medidas necessárias e suficientes para medi-lo. As atividades desta fase são descritas a seguir:

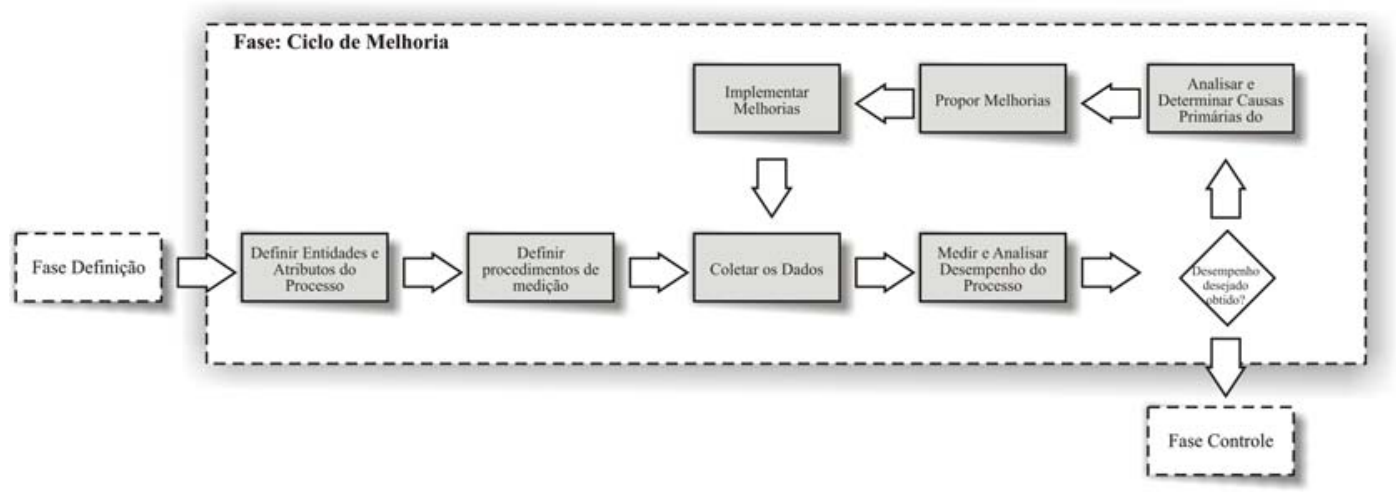

Figura 4 - Atividades da Fase Ciclo de Melhoria

Definir Entidades e Atributos do Processo - O objetivo dessa atividade é definir quais medidas serão necessárias para mensurar o desempenho do processo. Para cada processo do escopo deverão ser selecionados entidades e atributos que estejam relacionados às entradas e saídas do processo, isto é, atributos cujas medidas influenciam na determinação da qualidade do produto ou satisfazem os objetivos do processo.

Definir Procedimentos de Medição - O objetivo dessa atividade é a definição dos procedimentos de medição dos dados. Os procedimentos devem contemplar a determinação das medidas, responsáveis, a forma de coleta e armazenamento, análise, e comunicação e interpretação dos resultados. Qualquer problema nesses dados poderá afetar toda a análise e, conseqüentemente, seus resultados, podendo gerar informações distorcidas e prejuízo para a organização.

Coletar os Dados - Nesta atividade os dados registrados durante a execução dos processos devem ser coletados e validados pelo Analista de Medição. Depois de validados, os dados devem ser colocados sob baseline a fim de assegurar a integridade e o contexto atual do processo.

Medir e Analisar Desempenho do Processo - O objetivo desta atividade é medir e analisar o desempenho do processo através de ferramentas estatísticas aplicadas aos dados coletados. A partir da análise dos dados, o desempenho desejado do processo é definido de forma a atender as metas de desempenho estabelecidas para o processo em questão. Neste momento, a capacidade do processo atual é constatada observando o desempenho atual do processo frente aos limites de controle (desempenho desejado) especificados anteriormente. A capacidade pode ser verificada através de representação gráfica ou determinação dos índices de capacidade do processo ( $C p$ e $C p k$ ) (Florac e Carleton, 1999). Neste momento, pode ser oportuno reavaliar as metas de desempenho definidas para o processo a fim de obter metas mais realísticas, uma vez que o comportamento e desempenho do processo são conhecidos 
Analisar e Determinar Causas Primárias do Problema - Nesta atividade o Analista de Medição aplica um conjunto de ferramentas de análise de problemas para identificar detalhes do desempenho e variação do processo. Nesta ocasião é realizado um brainstorming, envolvendo o Dono do Processo, o Time de Trabalho e o Analista de Medição, com o objetivo de identificar possíveis causas primárias do baixo desempenho. É recomendado que neste momento seja utilizado o mapa do processo atual e diagramas de causa e efeito como apoio à atividade de investigação.

Propor Melhorias - O objetivo desta atividade é identificar pontos de melhoria de forma a resolver os problemas identificados. Para a auxiliar a resolução dos problemas, boas práticas referentes aos processos em questão podem ser obtidas através de benchmark de processos de outras organizações e relatos de experiência e através de modelos de referência da qualidade como CMMI, MPS.BR, ISO/IEC 15504 e literaturas específicas do processo. Neste momento, o mapa do processo é revisto e mudanças são propostas. Após a aprovação das mudanças, um plano de ação deve ser desenvolvido.

Implementar Melhorias - $\mathrm{O}$ objetivo desta atividade é implementar as melhorias propostas de acordo com o plano de ação. Este é o passo mais longo da abordagem e deve ser acompanhado para que sua execução seja garantida. Dificuldades podem ser encontradas durante a implementação das melhorias, portanto, é importante monitorar e re-planejar sempre que necessário para obter resultados efetivos.

\section{Fase 4: Controle}

A fase "Controle” é composta por duas atividades, como visualizado na Figura 5, e tem como objetivo o registro das lições aprendidas durante a melhoria do processo, bem como, realizar o controle e acompanhamento do processo melhorado no "Ciclo de Melhoria”.

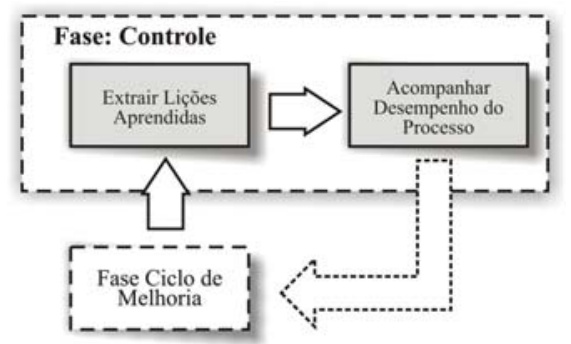

Figura 5 - Atividades da Fase Controle

Assim como na fase "Definição", os processos que estão sob controle na fase "Controle" devem ser revistos periodicamente, a fim de garantir que os processos prioritários estão sendo controlados. Essa revisão é importante uma vez que as prioridades dos processos mudam de acordo com a evolução estratégica da organização. As atividades desta fase são descritas a seguir:

Extrair Lições Aprendidas - O objetivo desta atividade é avaliar os resultados obtidos e registrar as lições aprendidas durante a melhoria do processo. Estas informações devem ser divulgadas para a organização.

Acompanhar Desempenho do Processo - Nesta fase o processo é acompanhado quantitativamente, visando garantir a manutenção do seu desempenho. Caso seja observado que o desempenho do processo regrediu a níveis não satisfatórios, este deve ser considerado como um processo a ser a melhorado na fase "Ciclo de Melhoria”. 


\section{Estudo de Caso}

A avaliação da abordagem proposta foi realizada através de um estudo de caso, o qual foi acompanhado pelos autores deste trabalho. A abordagem foi aplicada e observada durante aproximadamente seis meses em uma empresa de desenvolvimento de software, período no qual foram concluídos quatro Ciclos de Melhoria. A abordagem está sendo aplicada em mais uma organização, porém os resultados são ainda incompletos, por ela estar em uma fase inicial de implementação. Desta forma, apenas os dados da primeira organização serão apresentados neste artigo.

\subsection{Contexto da Organização}

O estudo de caso foi aplicado em uma organização de desenvolvimento de software que conta com aproximadamente 42 funcionários no seu setor de desenvolvimento. A organização tem como principal foco de atuação o desenvolvimento, evolução e comercialização de um produto de software do tipo ERP (Enterprise Resource Planning).

O ERP desenvolvido pela organização é composto de vários módulos, os quais sofrem evoluções constantes, gerando de tempos em tempos, novas versões do produto que são disponibilizadas à sua base de clientes. Embora este ERP seja considerado pela organização como um produto único, que atende a vários clientes, existem nele características que são customizáveis, a fim de atender a particularidade de cada cliente. Deste modo, o produto instalado em um cliente pode se apresentar de forma totalmente diferente do mesmo produto instalado em outro cliente, o que propicia uma variação significante da percepção de qualidade do produto pelo cliente. Em média, a organização libera uma nova versão do produto a cada um mês. Estas versões contemplam novos requisitos, alterações em requisitos já existentes, correções de bugs e, melhorias do produto em usabilidade, desempenho, segurança entre outras.

A empresa em questão já possuía iniciativas de melhoria do processo, já que era certificada ISO 9000 nos seus processos de produção, havia sido avaliada no nível G do MR-MPS e estava em processo de implementação dos processos do nível F.

\subsection{Aplicação da Abordagem}

A aplicação da abordagem Modus foi acompanhada pelos autores, aos quais couberam a responsabilidade de apresentar os conceitos e ferramentas que seriam utilizados e, realizar o treinamento inicial dos envolvidos no processo de melhoria da abordagem.

O estudo de caso é descrito a seguir apresentando um resumo das atividades realizadas em cada fase da abordagem Modus.

\section{Fase Iniciação}

Na fase inicial da aplicação da abordagem, foram identificadas as seguintes premissas dentro do contexto organizacional:

- Alinhar a aplicação da abordagem às outras iniciativas de melhoria da organização;

- Considerar a abordagem como apoio à implementação do processo de Medição do nível F do MPS.BR;

- Focar em melhorias pontuais dos processos críticos. 
A principal motivação para a utilização da abordagem Modus foi a possibilidade de atuar em processos críticos para os objetivos de negócio da organização, o que não é contemplada pela implantação do modelo MR-MPS, nos seus níveis mais baixos (até o nível B), uma vez que este pré-define os processos a serem melhorados.

Para manter o alinhamento com os projetos de melhoria executados na organização, a equipe do programa Modus foi formada pelos colaboradores que estavam diretamente envolvidos com o projeto MPS.BR, onde um desses colaboradores foi selecionado como Analista de Medição. Estas definições foram realizadas através de uma reunião e registradas em ata. A comunicação do inicio do projeto foi estabelecida por meio de um email enviado pela diretoria à todos os colaboradores da organização.

\section{Fase Definição}

Os objetivos de negócio da organização já estavam definidos e publicados em um portal interno da organização. Esses objetivos foram definidos com a utilização do método BSC (Balanced Scorecard) (Kaplan e Norton, 1992) e foram revistos no contexto dessa abordagem, contudo não foi observado necessidades de mudanças. Alguns dos objetivos de negócio da organização identificados foram:

- Aumentar o faturamento;

- Melhorar a satisfação do cliente;

- Aumentar a produtividade;

- Diminuir a geração de falhas nos produtos.

Os objetivos de negócio da organização foram decompostos em objetivos de melhoria. Os objetivos de melhoria inicialmente definidos foram:

- Melhorar o entendimento dos requisitos do cliente;

- Reduzir em aproximadamente 50\% o número de falhas identificadas pelo cliente;

- Reduzir o índice de retrabalho;

- Aumentar produtividade.

Com base nos objetivos de melhoria, os principais clientes foram identificados e suas necessidades levantadas. Como clientes foram considerados os clientes internos de cada um dos setores produtivos da empresa (comercial, implantação, desenvolvimento, suporte e homologação). Os requisitos destes clientes foram levantados em reuniões do tipo brainstorming. Alguns destes requisitos são apresentados a seguir:

- Requisitos melhor especificados;

- Documentação técnica do produto (diagrama de classe, casos de uso, etc);

- Agilidade para atender as necessidades do cliente (externo);

- Reduzir quantidade de bugs.

A empresa já possuía seus principais processos mapeados em função da ISO e MPS.BR, sendo necessário para a execução desta abordagem, apenas revisá-los. Dos processos mapeados foram selecionados os seguintes processos candidatos à aplicação do Ciclo de Melhoria, considerando os objetivos de melhoria e os requisitos do cliente:

- Processo de Especificação de Requisitos, e;

- Processo de Teste. 
Considerando as premissas para a implantação desta abordagem, restringiu se o foco da melhoria ao processo de teste, uma vez que o processo de especificação de requisitos era contemplado no escopo do MR-MPS e estava passando por constantes mudanças. Um fator importante a ser destacado e que contribuiu para identificação do processo de testes como crítico foram as afirmações dos gerentes e diretores, de que nos últimos meses a organização vinha alocando novos recursos para o setor de homologação - setor responsável pela a execução dos testes - contudo, o número de bugs escapados continuavam nos mesmos patamares. A meta definida para a melhoria do processo de teste foi:

- Reduzir o número de bugs escapados em $50 \%$ do valor.

Para o alcance desta meta, inicialmente foi planejado realizar melhorias num período de três meses (o que na realidade aconteceu em seis meses). Após as definições iniciou-se a fase Ciclo de Melhoria.

\section{Fase Ciclo de Melhoria}

Inicialmente buscou-se descrever em detalhes o processo de testes atual da organização, identificando todas as atividades, papéis, responsabilidades, entradas e saídas.

Observou-se que a organização já registrava algumas informações referentes à execução do processo de teste, embora estas não fossem efetivamente utilizadas. Deste modo, foi necessário rever as medidas e definir os procedimentos de coleta para as mesmas. As seguintes medidas foram definidas: módulo do produto ao qual o bug está associado; número de bugs por severidade (alto, médio, baixo); esforço de teste por módulo do produto; número de bugs encontrados pelo cliente (escapados) por módulo e; responsável pela abertura do bug.

O principal indicador definido para avaliação do desempenho do processo foi o DRE (Defect Removal Efficiency). Este indicador mostra a eficiência do processo de testes e é calculado da seguinte forma: $D R E=E /(E+D)$, onde $E$ é o número de bugs encontrados pelo processo de teste e, D é o número de bugs escapados. A Figura 6 apresenta o comportamento deste indicador no tempo, referente ao período anterior à implementação da melhoria.

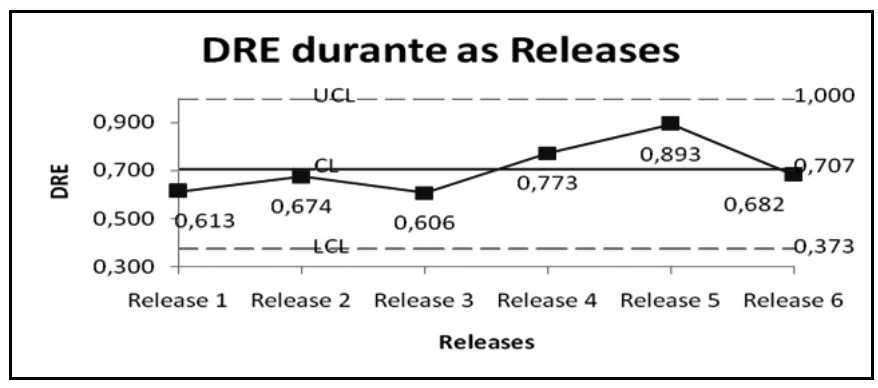

Figura 6 - Gráfico de Controle Antes da Melhoria

O objetivo definido para a melhoria foi fazer com que o DRE se aproximasse ao máximo de 1 (aumentando a capacidade do processo) e fosse estabilizado acima de 0.8 (diminuindo a variabilidade).

\section{Análise dos Dados}

A análise dos dados para a identificação das principais causas do baixo desempenho do processo foi realizada através de gráficos de Pareto. Os seguintes critérios foram 
utilizados para avaliação: número de bugs escapados por módulos, por tipo e por severidade e, o esforço médio de identificação do bug. Estas relações foram definidas a partir da análise dos dados coletados. Alguns exemplos das análises realizadas são apresentados na Figura 7.

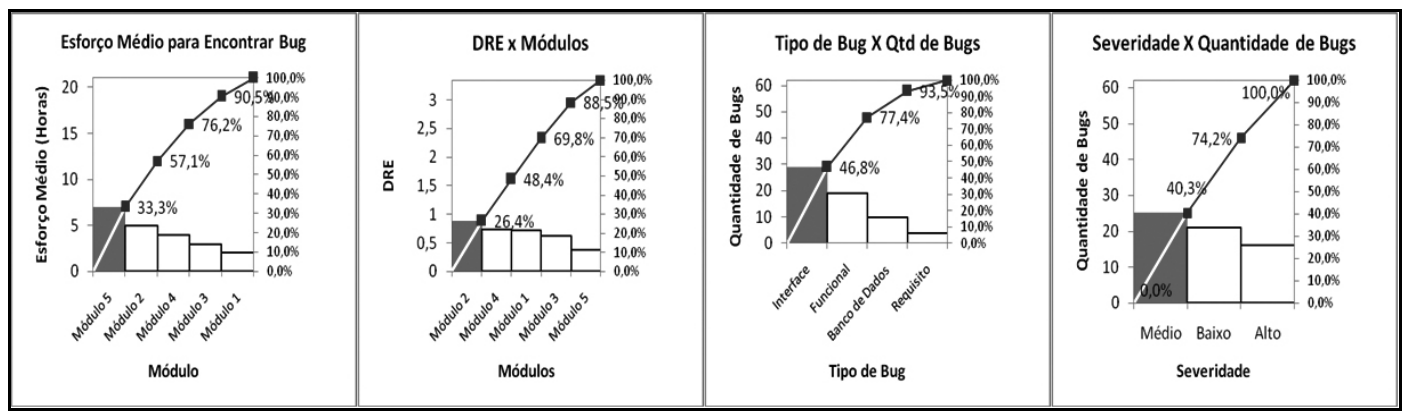

Figura 7 - Gráficos de Pareto com as Análises

Durante as análises, também se utilizou a ferramenta de Diagrama de Causa e Efeito, que foram elaborados através de brainstorming envolvendo a equipe de melhoria e os responsáveis pela execução do processo de testes. Com a análise, chegou-se a conclusão das prováveis causas do problema, as quais foram priorizadas para implementação da melhoria. A Tabela 1 mostra as principais causas identificadas.

Ações de melhoria foram propostas para cada causa primária identificada. Buscou-se implementar as ações pontuais de melhoria que fossem de rápida implantação e que não representasse grandes alterações no processo. Estas melhorias foram realizadas de forma integrada e alinhadas às ações de melhoria no contexto da implantação do nível F do MPS.BR. A Tabela 1 apresenta as causas raízes e as ações de melhoria implementadas.

Tabela 1 - Causas Primárias e Ações de Melhoria Determinadas

\begin{tabular}{|l|l|}
\hline \multicolumn{1}{|c|}{ Causas raízes } & \multicolumn{1}{c|}{ Ações de melhoria } \\
\hline $\begin{array}{l}\text { Deficiência nos Projetos de } \\
\text { Teste. }\end{array}$ & $\begin{array}{l}\text { •Treinamento da equipe em técnicas de Projeto de Teste. } \\
\text { •Adoção das práticas de revisões em Projetos de Teste. }\end{array}$ \\
\hline $\begin{array}{l}\text { Complexidade de determinados } \\
\text { módulos. }\end{array}$ & $\begin{array}{l}\text { •Elaboração de testes mais robustos, especialmente com Projeto de Teste mais } \\
\text { detalhado, nos módulos críticos. } \\
\bullet \text { Envolvimento da equipe de Analista de Sistema nas revisões dos projetos de } \\
\text { teste. }\end{array}$ \\
\hline $\begin{array}{l}\text { Dificuldades em definir suíte de } \\
\text { teste para regressão parcial. }\end{array}$ & $\begin{array}{l}\text { •Desenvolver em conjunto com a equipe de desenvolvimento, documentação de } \\
\text { correlação entre as principais funcionalidades do sistema. }\end{array}$ \\
\hline $\begin{array}{l}\text { Falta de validação dos } \\
\text { requisitos com o cliente. }\end{array}$ & $\begin{array}{l}\text {-Alteração no processo de requisitos, introduzindo a prática de obter aceite do } \\
\text { cliente. }\end{array}$ \\
\hline
\end{tabular}

Com a implementação destas ações de melhoria obteve-se a meta esperada para o desempenho do processo, após o quarto ciclo de melhoria, como podemos observar no gráfico de controle da Figura 8. O indicador DRE se aproximou de 1, estabilizando-se entre 0.8 e 0.9, o que representou uma redução significativa no número de bugs escapados. Com o objetivo de garantir a estabilidade do processo após o projeto foi estabelecido o uso de gráficos de controle que serão analisados freqüentemente pela organização. 


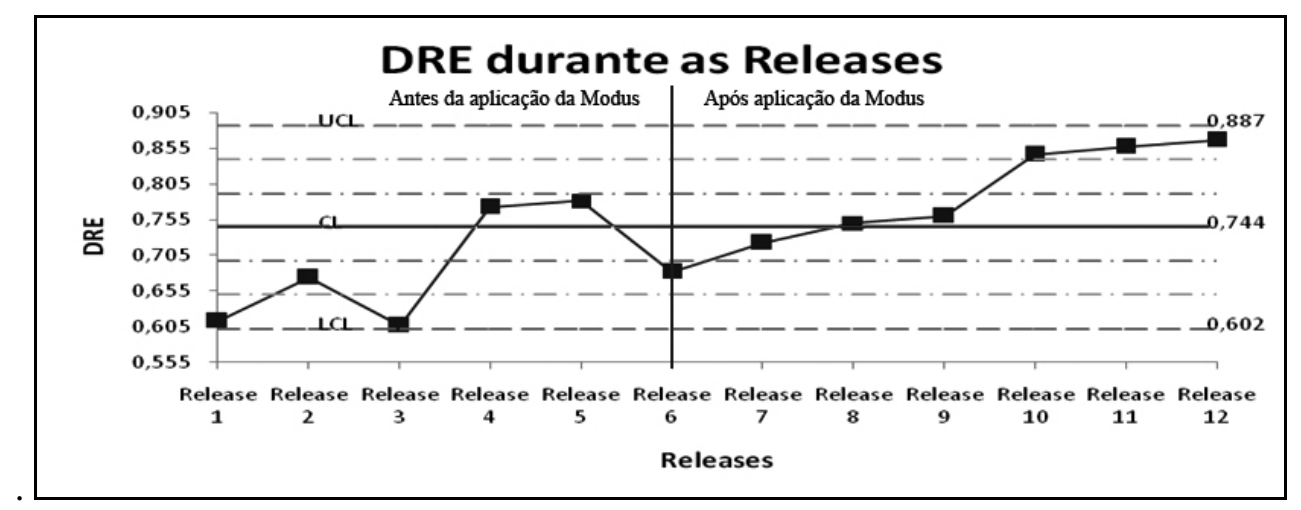

Figura 8 - Evolução do DRE

\section{Fase Controle}

Esta fase envolveu a implementação do controle do processo de testes, que já havia sido iniciado na etapa anterior. Foi realizado um brainstorming entre os envolvidos no projeto, incluindo representantes da gerência e diretoria, onde foram discutidas as principais dificuldades, lições aprendidas e retornos obtidos, os quais foram registrados na base de conhecimento da organização.

\subsection{Análise da Aplicação}

Algumas dificuldades foram encontradas na implantação da abordagem, especialmente no que se refere ao entendimento dos objetivos de negócio da organização, como mensurá-los e relacioná-los à melhoria dos processos. Acredita-se que o baixo nível de maturidade da organização tenha contribuído para tal dificuldade.

Durante a aplicação da abordagem pontos positivos e negativos foram observados e analisados. Como ponto positivo pode-se destacar a forma de planejamento do Ciclo de Melhoria considerando utilizar ciclos curtos, com aproximadamente um mês de duração. Outros pontos identificados foram a falta de uma representação mais amigável para a abordagem e ferramentas de apoio. Para atender estas questões representamos a abordagem por meio de uma notação de processo e disponibilizamos em um site web $^{3}$, juntamente com alguns templates e planilhas de apoio desenvolvidas no Microsoft Excel.

Outro ponto relevante identificado foi que algum tempo depois da finalização da fase Ciclo de Melhoria (durante a fase Controle), a empresa observou que mesmo com o atendimento às necessidades de melhoria, as necessidades do cliente não estavam sendo atingidas. Isso motivou novas análises em relação à meta de desempenho para o processo. Foi observado que o indicador de DRE, como definido durante a implementação da abordagem, não atendia às necessidades de todos os clientes, dessa forma, a definição do indicador foi revista. A empresa passou a considerar o DRE por cliente, uma vez que o impacto do bugs está associado ao mesmo. Para a empresa era importante que o DRE fosse extremamente baixo quando considerado alguns clientes, enquanto que, para os demais, valores mais elevados do DRE eram aceitáveis. Tal situação fez com que o processo de teste voltasse a ser objeto de melhoria.

\footnotetext{
${ }^{3}$ Disponível em: http://www.cin.ufpe.br/ processos/modus
} 


\section{Conclusões}

De forma geral, este trabalho aponta para a viabilidade de se utilizar os conceitos de medição, como ferramenta de apoio à melhoria dos processos de uma organização de desenvolvimento de software, mesmo sendo uma organização com baixo nível de maturidade. Com a aplicação desta abordagem pode-se obter resultados significativos e mensuráveis relativos à melhoria do desempenho do processo crítico, selecionado para o escopo da melhoria.

Um ponto importante é que a abordagem permite com que os esforços de melhoria sejam realizados de acordo com a disponibilidade de recursos de cada organização, uma vez que, o escopo da melhoria é definido pela própria organização. Desta forma, organizações que tem restrições de recursos podem realizar a melhoria em escopo reduzido, possibilitando que se trabalhe apenas um processo.

Notamos que esta abordagem pode ser utilizada como uma forma complementar à implantação de modelos de melhoria e, ainda sugerimos sua utilização no início dos programas de melhoria, quando ainda se está trabalhando os níveis mais baixos de medição.

Observou-se que ainda existe uma grande dificuldade e resistência nas empresas na aplicação de métodos de medição, devido ao receio de que estes métodos fossem de difícil entendimento. Ao mesmo tempo, pudemos concluir que é possível obter resultados significativos com a utilização de ferramentas simples e conhecidas.

A abordagem apresentada poderá vir a ajudar as organizações na implementação de um programa de melhoria a fim de melhorar os seus processos de desenvolvimento e, assim, contribuir para a melhoria de produtos de software gerados. Além disso, a abordagem provê uma forma mais flexível para as organizações implementarem melhoria de seus processos sem a imposição dos formalismos considerados nos modelos de referência.

\section{Referências}

Associação para a Promoção da Excelência do Software Brasileiro - SOFTEX. (2007) “MPS.BR - Guia Geral, v1.2”. 2007. Disponível em: <http://www.softex.br>.

Chrissis, M. D., Konrad, M. e Shrum S. (2007) "CMMI: guidelines for process integration and product improvement”. Addison-Wesley. 2007.

Deming, W. E. (1986) "Out of the Crisis”. MIT Center for Advanced Engineering Study, Cambridge, MA. 1986.

Dybã, T. (2003) "Factors of Software Process Improvement Success in Small and Large Organizations: An Empirical Study in the Scandinavian Context”. ESEC/FSE, September, 2003.

Dybã, T. (2005) “An Empirical Investigation of the Key Factors for Success in Software Process Improvement”. IEEE Transactions On Software Engineering, v. 31, n. 5, May 2005.

Eckes, G. “Six Sigma for Everyone”. John Wiley \& Sons, Inc. 2001.

Florac, W. A. e Carleton, A. D. (1999) "Measuring the Software Process: Statistical Process Control for Software Process Improvement”. Addison-Wesley. 1999. 
Goethert, W. e Hayes, W. (2001) "Experiences in Implementing Measurement Programs”. Pittsburgh: Carnegie Mellon University - Software Engineering Institute. 2001.

Harry, M. e Schroeder, R. "Six Sigma: The Breakthrough Management Strategy Revolutionizing the World’s Top Corporations”. Currency. 1998.

Humphrey, W. S. (1995) “Introducing the personal software process". Springer Netherlands: Annals of Sofware Engineering, v. 1, n. 1, p. 311-325. 1995

ISO/IEC 15504. (2003) "ISO/IEC 15504 - Information Technology - Process Assessment, International Standard (IS)”. 2003.

Juran, J. M. (1988) “Juran's Quality Control Handbook”. McGraw-Hill. 1988.

Kaplan, R. S. e Norton, D. P. (1992) “The Balanced Scorecard: Measures That Drive Performance”. Harvard Business Review. 1992.

NBR-ISO 9000. (2000) “ISO9000:2000 - Sistemas de gestão da qualidade: Fundamentos e vocabulário”. Rio de Janeiro: ABNT. Dezembro de 2000.

NBR-ISO/IEC 12207. (1998) "IEC 12207: Tecnologia da Informação - Processos de Ciclo de Vida do Software”. Rio de Janeiro: ABNT. 1998.

Pande, P. S., Neuman, R. P. e Cavanagh, R. R. “The Six Sigma Way: How GE, Motorola, and Other Top Companies Are Honing Their Performance”. McGraw-Hill. 1998.

Rocha, A. R. C., Maldonado, J. C. e Weber, K. C. (2001) "Qualidade de Software: Teoria e Prática”. São Paulo: Makron. 2001.

Salviano, C. F. (2006) "Melhoria e Avaliação de Processo de Software com ISO/IEC 15504-5:2006”. Lavras: UFLA/FAEPE. 2006. (Publicação do Curso de Pósgraduação "Latu Sensu” (Especialização) à Distância em Melhoria de Processo de Software).

Santana, A. F. L. (2007) "Problemas em Iniciativas de Melhoria de Processos de Software sob a Ótica de uma Teoria de Intervenção”. Dissertação (Mestrado) Universidade Federal de Pernambuco, Recife, 2007.

Serrano, M. A. , Oca, C. M. e Cedillo, K (2006) “An Experience on Implementing the CMMI in a Small Organization Using the Team Software Process”. In: Garcia, S., Graettinger, C. e Kost, K. Proceedings of the First International Research Workshop for Process Improvement in Small Settings. Pittsburgh: Carnegie Mellon - Software Engineering Institute. 2006.

Shewhart, W. A. (1931) "Economic control of quality of manufactured product”. New York: D. Van Nostrand Company, 501 p. 1931.

Siviy, J. M., Penn, M. L. e Stoddard, R. W. (2008) “CMMI and Six Sigma: Partners in Process Improvement”.

Tayntor, C. B. (2003) “Six Sigma Software Development”. Florida: Auerbach. 2003. 